\title{
Does prior sepsis alter subsequent circadian and sickness behaviour response to lipopolysaccharide treatment in mice?
}

\author{
Sean T. Anderson • Emma K. O'Callaghan • \\ Sean Commins • Andrew N. Coogan
}

Received: 22 October 2013/Accepted: 19 November 2013/Published online: 15 December 2013

(C) Springer-Verlag Wien 2013

\begin{abstract}
Previous data has shown that prior history of immune challenge may affect central and behavioural responses to subsequent immune challenge, either leading to exaggerated responses via priming mechanisms or lessened responses via endotoxin tolerance. In this set of experiments we have examined how previously lipopolysaccharide (LPS)-induced sepsis shapes the response to subsequent treatment with lower dose LPS. After treatment with LPS $(5 \mathrm{mg} / \mathrm{kg}$ ) or saline mice were allowed to recover for 3-4 months before being challenged with a lower dose of LPS $(100 \mu \mathrm{g} / \mathrm{kg})$ for assessment of sickness behaviours. Performance on the open field test and the tail suspension test was assessed, and no evidence was found that prior sepsis altered sickness or depressive-like behaviour following LPS treatment. We then examined the responsiveness of the circadian system of mice to LPS. We found that in control animals, LPS induced a significant phase delay of the behavioural rhythm and that this was not the case in post-septic animals (4-6 weeks after sepsis), indicating that prior sepsis alters the responsivity of the circadian system to subsequent immune challenge. We further assessed the induction of the immediate early genes c-Fos and EGR1 in the hippocampus and the suprachiasmatic nucleus (SCN; the master circadian pacemaker) by LPS in control or post-septic animals, and found that post-septic animals show elevated expression in the hippocampus but not the SCN. These data suggest that previous sepsis has
\end{abstract}

S. T. Anderson and E. K. O'Callaghan contributed equally to the work.

S. T. Anderson · E. K. O'Callaghan · S. Commins ·

A. N. Coogan $(\bowtie)$

Department of Psychology, National University of Ireland

Maynooth, County Kildare, Ireland

e-mail: Andrew.Coogan@nuim.ie some effect on behavioural and molecular responses to subsequent immune challenge in mice.

Keywords LPS · Sepsis · Circadian · Depression · Neuroimmune · Anxiety

\section{Introduction}

Peripheral administration of the gram-negative bacterial endotoxin lipopolysaccharide (LPS) has long been recognised to produce a syndrome of behavioural changes termed sickness behaviour, including decreased locomotion and exploration, decreased food intake and increased sleep, as well as inducing increased depressive-like behaviours (McCusker and Kelley 2013). This behaviour change may reflect an adaptive response to sickness that best allows an organism to fight off infection (Dantzer 2001). It is believed that both peripheral neural activation and production of humoral factors in response to the immune challenge leads to induction of pro-inflammatory mediators in the brain, and it is the action of these mediators (e.g. TNF- $\alpha$, IL-1 $\beta$ ) that underpin the behavioural changes elicited by immune challenge (Dantzer et al. 2008).

A homeostatic control system that may be of importance in sickness behaviour is the circadian system (Coogan and Wyse 2008). Circadian rhythms are recurring patterns in a wide spectrum of physiological, endocrine, behavioural and cognitive parameters that recur with periods of approximately $24 \mathrm{~h}$ (Mohawk et al. 2012). The circadian timekeeping network functions as a network of connected central and peripheral oscillators, with what is thought of as a master circadian pacemaker located to the suprachiasmatic nucleus (SCN) of the anterior hypothalamus 
(Dibner et al. 2010). The correct functioning of the circadian system is increasingly being recognised as being a key contributor to health and well being, and circadian dysfunction is linked with numerous metabolic, neurological and psychiatric disorders (Bass and Takahashi 2010; Coogan et al. 2013; McClung 2013). An area that may be of importance in the modulation of the circadian system during pathologies is the neuroimmune regulation of central circadian pacemakers (Coogan and Wyse 2008). Intriguingly, from a sickness behaviour point of view, there is circadian control over the affected behavioural systems, such as sleep, locomotor control and mood (McClung 2013; Coogan and Wyse 2008).

The nature of the behavioural response elicited by a peripheral immune challenge may be determined by the background against which that challenge is presented. For example, LPS treatment of older animals produces exaggerated sickness behaviour response when compared to younger animals (Henry et al. 2009). Further, ongoing neuroinflammation and/or neurodegeneration "primes" behavioural and central responses to immune challenge, and this may be an important mechanism in the pathophysiology of delirium (Griffin et al. 2013; Cunningham 2013). Thus, previous neuroimmunological history may impact behavioural responses to subsequent immune challenge and central responsiveness to inflammatory mediators, and these effects may be pertinent in a wide range of clinical conditions. One such condition, of profound clinical importance, is sepsis. Sepsis is a syndrome characterised by systemic inflammation that is associated with significant mortality (Angus and van der Poll 2013). Survivors of sepsis have been described as exhibiting an ongoing syndrome termed post-septic encephalopathy, in which there are ongoing cognitive difficulties (Semmler et al. 2013; Iwashyna et al. 2010). Similar findings of changes in behavioural and cognitive parameters, that are also accompanied by a chronic neuroinflammation, have been described in animal models (Qin et al. 2007; Weberpals et al. 2009; O'Callaghan et al. 2012). Given the persistent upregulation of central inflammation following sepsis, we set out to enquire whether prior sepsis would impact on both behavioural and central molecular response to peripheral LPS treatment.

\section{Materials and methods}

\section{Animals}

For the purpose of all experiments male C57BL/6 mice (Charles River, Kent, UK) aged between 8 and 16 weeks were used $(N=61$ in total). Animals were group housed in a 12:12 light:dark cycle for 2 weeks prior to LPS administration. Food and water were available ad libitum and temperature was $21 \pm 1{ }^{\circ} \mathrm{C}$ and humidity was $50 \pm 10 \%$. Unless housed in isolation for circadian wheel running experiments, animals remained housed in groups of 2-4 in polypropylene cages $(33 \mathrm{~cm}$ long $\times 15 \mathrm{~cm}$ wide $\times 13 \mathrm{~cm}$ high) with wood chip bedding and environmental enrichment (shredded paper and cardboard tubes). All procedures were approved by the Research Ethics Committee, National University of Ireland Maynooth, and were licensed by the Department of Health and Children, Ireland under statutory instrument (SI) No. 543 of 2012 and the European directive 2010/63/EU.

\section{LPS-induced sepsis}

All saline and LPS injections were made up fresh on the treatment day, and given intraperitoneally in a final injection volume of $0.125 \mathrm{ml} .0 .9 \%$ sterile saline was made up fresh for control injections, while LPS (serotype 0111.B4, Sigma Ireland; Qin et al. 2007) was made up to a $5 \mathrm{mg} / \mathrm{kg}$ dose in sterile saline. Injections were given intraperitoneally (i.p.) between zeitgeber time (ZT) 06 and 08, where ZT0 is defined as the time of lights on. Animals were allowed to recover for at least 1 month prior to behavioural testing. Mortality and significant moribundity requiring euthanasia occurred within approximately $7 \%$ of animals $(N=3)$ following the induction of sepsis.

Assessment of behaviour on the open field test and the tail suspension test

Between 3 and 4 months after the septic LPS/saline treatment, mice were treated in a counterbalanced fashion ( 2 weeks between treatments) with either saline or LPS (i.p; $100 \mu \mathrm{g} / \mathrm{kg}$ ) injected at ZT2. Two hours after this treatment animals were tested on the open field test (OFT) and $9 \mathrm{~h}$ after treatment animals were tested on the tail suspension test (TST), as depressive-like behaviours develop over a longer period following peripheral LPS treatment compared to sickness behaviour (Dantzer et al. 2008). For the OFT, testing occurred in an arena with a diameter of $100 \mathrm{~cm}$ (light intensity of 200 lux in the centre of the arena). The primary outcome of the OFT was to assess sickness behaviour in terms of locomotor behaviour, with anxiety related outcomes (time spent in centre vs. time spent in the periphery) secondary endpoints. Each animal was placed in the centre of the arena and allowed to explore for $300 \mathrm{~s}$ during which their distance moved, velocity in $\mathrm{cm}^{2}$ and time spent moving were automatically tracked with Ethovision 3.1 software (Ethovision 3.1; Noldus Information Technology, Leesburg, VA). The percentage of time spent in the inner $50 \%$ and outer $50 \%$ of the arena were also measured. For the TST, mice were 
removed from the home cage and attached to a support raised $121 \mathrm{~cm}$ above a cage containing woodchip bedding by tape placed $1 \mathrm{~cm}$ from the tip of their tales for $6 \mathrm{~min}$. Mice were suspended for $6 \mathrm{~min}$ each, with immobility being recorded throughout the entire $6 \mathrm{~min}$. Immobility was defined as the complete absence of movement (Steru et al. 1985).

Assessment of circadian rhythms in wheel running behaviour

For the purpose of behavioural monitoring of circadian rhythms, animals were individually housed in polypropylene cages $(33 \mathrm{~cm}$ long $\times 15 \mathrm{~cm}$ wide $\times 13 \mathrm{~cm}$ high) equipped with steel running wheels $(11.5 \mathrm{~cm}$ diameter) with food and water available ad libitum. The light source was standard fluorescent light bulbs with an average 150 lux luminance level in each individual cage. Cages were ventilated via axial fans to prevent the build-up of pheromones and these fans produced white noise at the level of $50 \mathrm{~dB}$. In order to establish whether an altered behavioural response would be seen in post-septic animals compared to controls following a second immune challenge, 14 days after induction of sepsis or control treatment (either $5 \mathrm{mg}$ / $\mathrm{kg}$ LPS or saline), animals were placed into constant darkness (DD) for 14 days and allowed to free run. Animals then received either i.p. saline or an i.p. injection of LPS at a dose of $100 \mu \mathrm{g} / \mathrm{kg}$ at CT15. This time was chosen as it previously has been shown administration of low dose LPS at this phase induces photic like phase delays of locomotor activity (Marpegán et al. 2005). Following treatment, the animals were maintained in DD for an additional 14 days before receiving a counterbalanced treatment of either LPS or saline at CT15. Following this treatment, the animals' activity patterns in DD were followed for 14 days and phase shifts of locomotor activity rhythms assessed. The line of best fit method was used to assess the phase shift magnitudes from the actogram data, fitting the line through activity onsets 7 days before and 10 days after treatment with LPS $100 \mu \mathrm{g} / \mathrm{kg}$ or vehicle. The differences between the lines from the actograms were rated by two independent researchers blind to the experimental procedure. Following each CT15 treatment, the circadian parameters of free running period and rhythm amplitude were calculated for each animal using the Chronobiology Kit Chi Squared procedure.

Immediate early gene expression and markers of microglia

In order to establish whether post-septic animals would exhibit altered immediate early gene expression following LPS treatment compared to controls, 6 week old male mice were group housed in colonies of three in cages equipped with appropriate environmental enrichment and habituated to a 12:12 LD cycle for 2 weeks (150 lux, lights on 0500 hours) prior to experimentation. The animals were treated with either LPS $5 \mathrm{mg} / \mathrm{kg}$ i.p. to induce sepsis, or saline. The animals were allowed to recover following the induction of sepsis and were maintained for 3-4 months under a 12:12 LD cycle. Both post-septic and saline treated controls then received an i.p. injection of LPS at a dose of $100 \mu \mathrm{g} / \mathrm{kg}$ at ZT1-2. Animals were then terminally anaesthetized and perfused transcardially with $4 \%$ paraformaldehyde. Animals were perfused 2, 4 or $9 \mathrm{~h}$ after LPS treatment. Timepoints at 2 and $9 \mathrm{~h}$ were used for assessment of immediate early gene expression, whilst those at $4 \mathrm{~h}$ were used to assess microglial markers. We examined $4 \mathrm{~h}$ as there has been reports of changes in microglial function $4 \mathrm{~h}$ after LPS treatment (e.g. Chen et al. 2008; Henry et al. 2009), and the 2 and 9 h timepoints to coincide with the times of behavioural testing. We examined the hippocampus, as sickness behaviour has been associated in a number of studies with neurochemical changes in this brain region (e.g. Frenois et al. 2007) and we examined the SCN given its profound importance in circadian timekeeping (Dibner et al. 2010). Another group of animals was treated with LPS 1 month after sepsis treatment and sampled $4 \mathrm{~h}$ after LPS and these animals' brains were later used for the assessment of microglial markers.

Following cryoprotection in $30 \%$ sucrose brains were coronally sectioned ( $30 \mu \mathrm{m}$ sections) on a freezing sliding stage microtome (Leica, Germany) and then sections processed according to a standard avidin-biotin complex nickel DAB immunohistochemical protocol (see Beynon and Coogan 2010). All sections processed for the same antigens were processed in parallel across experimental groups. The primary antibodies used in this study were: c-Fos, Santa Cruz Biotechnology (sc-52), rabbit polyclonal, used at 1:2,000; EGR1, Santa Cruz Biotechnology, rabbit polyclonal (sc-189), 1:3,000; Cd-11b, ABD Serotec (MCA74GA), rabbit polyclonal, used at 1:1,000; F4/80, ABD Serotec (MCA497GA), rabbit polyclonal used at 1:100 and IL-1 $\beta$ Peprotech (500 P-51), rabbit polyclonal used at 1:50.

Photomicrographs of hippocampus and SCN were taken using a digital camera connected to an Olympus BX-51 light microscope equipped with an image analysis digital system (ImageJ 1.43, NIH, USA). All images were taken using the same camera and magnification settings. For analysis, brain sections were examined under either the $10 \times$ or $40 \times$ objective lens. Between 3 and 6 bilateral images were evaluated for each individual animal and region depending on the area being examined and a mean value obtained for each animal for each region. Only 
sections from the mid rostrocaudal level of the SCN were examined (hence limiting the number of sections available from any one animal) and for the hippocampus 4 to 6 bilateral sections per animal of the medial dorsoventral axis of the hippocampus were analysed. Immunoreactive cells in each region of interest were quantified using quantification of immunoreactive (ir) cell number by an observer by by-eye counting for the purpose of analysing immunoreactive nuclear staining for c-Fos and EGR1. The observer was blinded to the experimental procedure during optical density measurements or quantification of immunoreactive cells. A previously described method for assessing glia whereby the image was binarised for analysis was used for integrated optical density measurements (Vilaplana and Lavialle 1999) was used to assess staining levels for Cd11b, F4/80 and IL-1 $\beta$.

\section{Statistics}

All data values given are mean \pm SEM. Inferential statistical analysis was via factorial between groups or mixed between-within groups ANOVAs and $t$ tests as appropriate (paired or independent). $P<0.05$ was deemed statistically significant. Where multiple comparisons were carried out the appropriate Bonferroni correction was applied.

\section{Results}

Mice tested on the OFT showed decreased exploration (as assessed by time spent mobile, distance travelled and velocity) following LPS treatment, irrespective of whether they had previously undergone sepsis or not (Fig. 1a; $P<0.001$ for each dependent variable for main effect of acute LPS). However ANOVA reveals no significant sepsis effect or sepsis $\times$ LPS interactions on measures of distance travelled, time spent mobile or velocity for any of the dependent variables analysed. When areas of the open field explored were analysed we found there was a significant difference in exploration of the periphery versus the centre, with animals spending less time in the centre following LPS than saline treatment $\left(F_{1,14}=7.4, P<0.05\right.$ for main effect of acute LPS on time spent in the centre). However there was no effect of prior sepsis nor a sepsis $\times$ LPS treatment interaction $(P=0.31$ and $P=0.39$ respectively) indicating that prior sepsis did not alter the extent to which animals exploration of the open field was altered following an acute peripheral LPS challenge. When examined on the TST, both animals that had previously undergone sepsis and those that had not showed increased immobility following LPS compared to saline treatment $\left(F_{1,14}=17\right.$, $P<0.001$ for main effect of acute LPS treatment; Fig. 1c). However there was no interaction effect of prior sepsis on
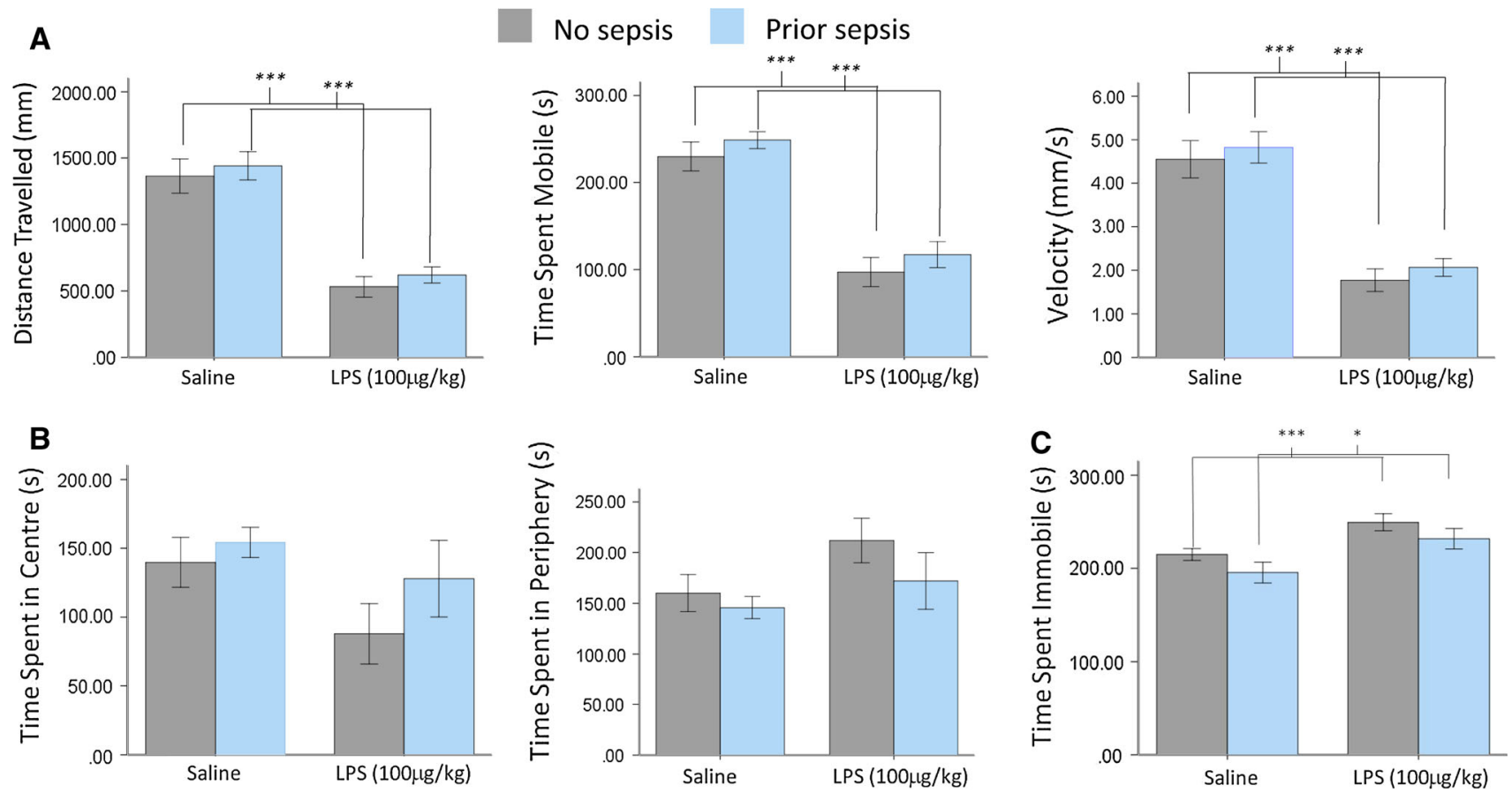

Fig. 1 Responses of post-septic and control mice on performance in the open field and tail suspension tests. a Responses to both saline and LPS treatment $(100 \mu \mathrm{g} / \mathrm{kg}$, i.p.) on locomotor parameters in the open field test in both post-septic and control animals; b time spent exploring the periphery and centre of the open field test after acute
LPS treatment in control and post-septic animals; c time spent immobile on the tail suspension test following saline and LPS treatment in control and post-septic animals. $N=7$ for the post-septic group and $N=9$ for the control group. $* * * P<0.001, * * P<0.01$, $* P<0.05$ 
the immobility subsequently induced by LPS treatment $(P=0.65)$ and pairwise comparisons indicate that LPS induces significantly more immobility than saline treatment in both control and post-septic animals. Therefore the results from these experiments indicate that prior sepsis does not affect behavioural responses to a $100 \mu \mathrm{g} / \mathrm{kg}$ treatment of LPS as measured on the OFT and the TST.

We analysed immediate early gene expression at 2 and $9 \mathrm{~h}$ following LPS treatment in post-septic and control animals. In the hippocampus there were significant main effects of sepsis on c-Fos expression in the dentate gyrus and the CA1 $\left(F_{1,15}=6.47, P<0.05\right.$ and $F_{1,15}=7.01$, $P<0.05$ respectively). Pairwise analysis reveals a significant upregulation of c-Fos in the dentate gyrus at $2 \mathrm{~h}$ in post-septic animals and a decrease in the CA1 in postseptic animals $9 \mathrm{~h}$ after LPS treatment (Fig. 2a, b). When EGR-1 was examined, there was a main effect of sepsis on EGR1 expression following acute LPS treatment in the dentate gyrus and the CA1 $\left(F_{1,15}=7.7, P<0.05\right.$ and $F_{1,15}=4.9, \quad P<0.05$ respectively). Pairwise analysis reveals EGR1 expression at $2 \mathrm{~h}$ following LPS treatment in the CA1 was enhanced in post-septic animals compared to controls (Fig. 2c, d). We then examined microglial marker expression in both post-septic (1 month after sepsis) and control animals following a peripheral LPS challenge. In the hippocampus we observed that expression of both $\mathrm{Cd}$ $11 \mathrm{~b}$ and F4/80 was higher in post-septic animals than in controls (Fig. 3). When expression of the pro-inflammatory cytokine IL- $1 \beta$ was examined there was no difference in its expression in the hippocampus between post-septic animals and controls following LPS treatment at either 2 or $9 \mathrm{~h}$ (Fig. 4).

When tested for phase shifts of their circadian rhythms in response to LPS treatment, animals that had previously not undergone sepsis showed a moderate phase delay of the free running rhythm after treatment with LPS at CT15 $(-0.68 \pm 0.14 \mathrm{~h}$ for LPS vs. $-0.10 \pm 0.07 \mathrm{~h}$ for saline treatment; $P<0.05$; Fig. 5). There was no change in free running period or rhythm power following LPS treatment, nor any differences between the post-septic and control groups (data not shown). For post-septic animals LPS treatment did not elicit a significant phase shift compared to control saline treatment $(-0.22 \pm 0.08 \mathrm{~h}$ for LPS treated animals compared to $-0.25 \pm 0.13 \mathrm{~h}$ for saline treatment). ANOVA revealed a main effect for shift magnitude elicited by the LPS $\left(F_{1,20}=6.79, P<0.05\right)$ and an interaction effect for prior sepsis and acute LPS $\left(F_{1,20}=8.65, P<0.01\right)$.

There were no differences in the expression of c-Fos or EGR-1 in the SCN of post-septic animals compared to controls following LPS treatment (Fig. 6) and further there was also no difference in either $\mathrm{Cd}-11 \mathrm{~b}$ or F4/80 expression after LPS treatment in the SCN of control and postseptic animals (Fig. 7).
A
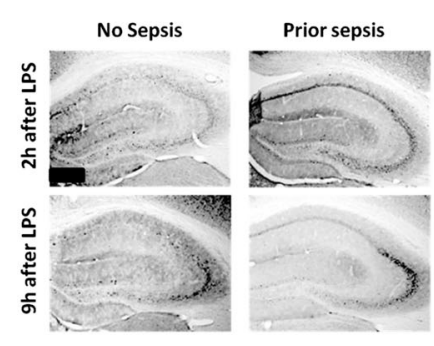

B

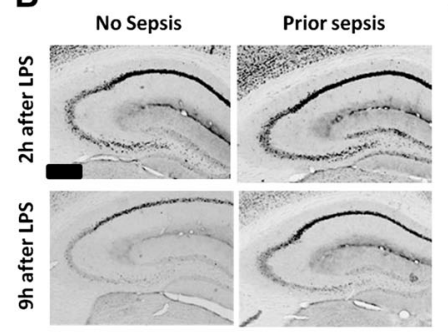

C

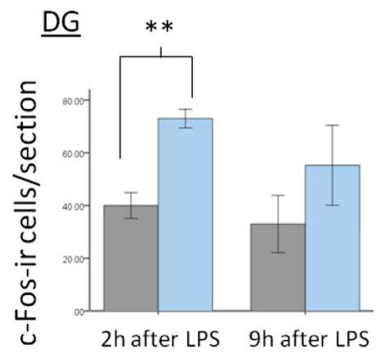

D

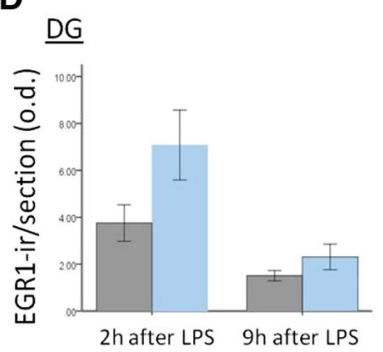

Fig. 2 Effect of prior sepsis on the expression of immediate early gene products in the hippocampus of control and post-septic animals. a and b Representative photomicrographs showing c-Fos and EGR1 immunostaining respectively in the hippocampus in control and postseptic animals at 2 and $9 \mathrm{~h}$ after a LPS injection. $N=4-5$ for each
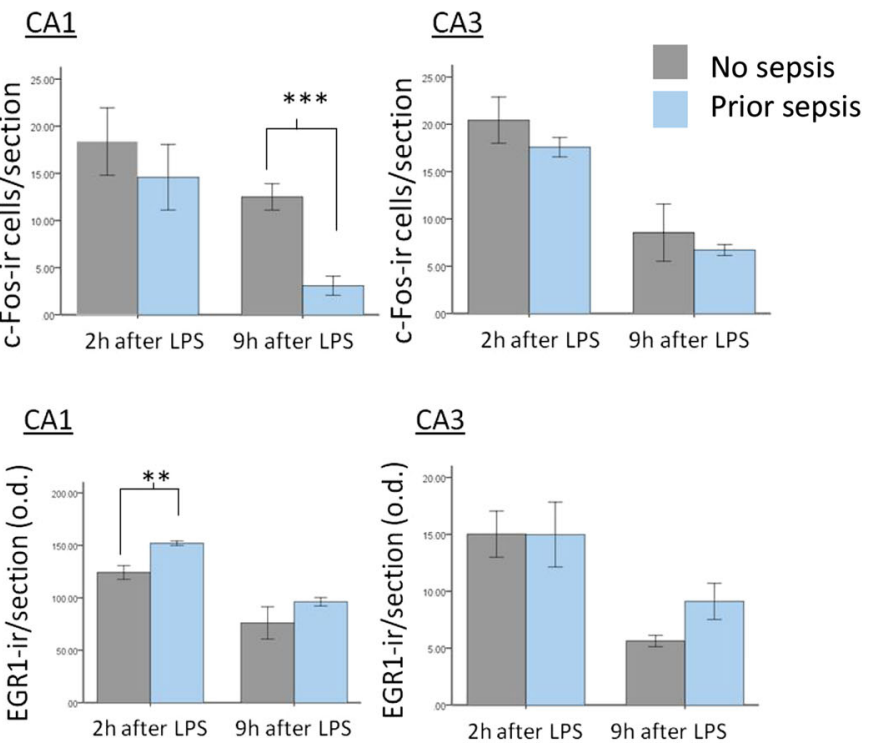

group per timepoint. Scale bar $300 \mu \mathrm{m}$. c and d Quantification of c-Fos and EGR1 immunostaining respectively in hippocampal subfields at 2 and $9 \mathrm{~h}$ after acute LPS treatment. $* * * P<0.001$, $* * P<0.01, * P<0.05$ 
Fig. 3 Expression of microglial markers in the hippocampus after LPS treatment of both control and post-septic animals. a Sample photomicrographs of Cd-11b immunostaining and its quantification in the hippocampus $4 \mathrm{~h}$ after LPS treatment in both control and post-septic animals; b sample photomicrographs of F4/80 immunostaining and its quantification in the hippocampus $4 \mathrm{~h}$ after LPS treatment in both control and post-septic animals. $N=7-8$ pre group. Scale bars $300 \mu \mathrm{m}$. $* P<0.05$

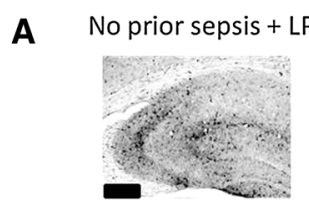

Prior sepsis + LPS
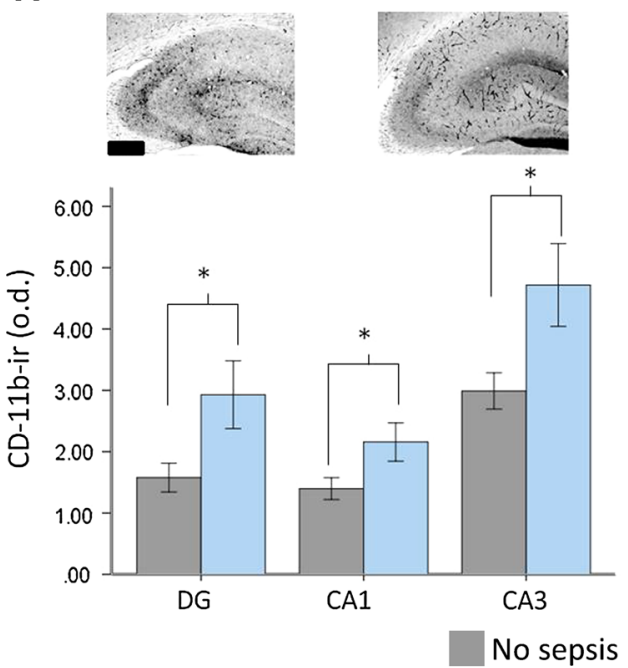

B No prior sepsis + LPS

Prior sepsis + LPS
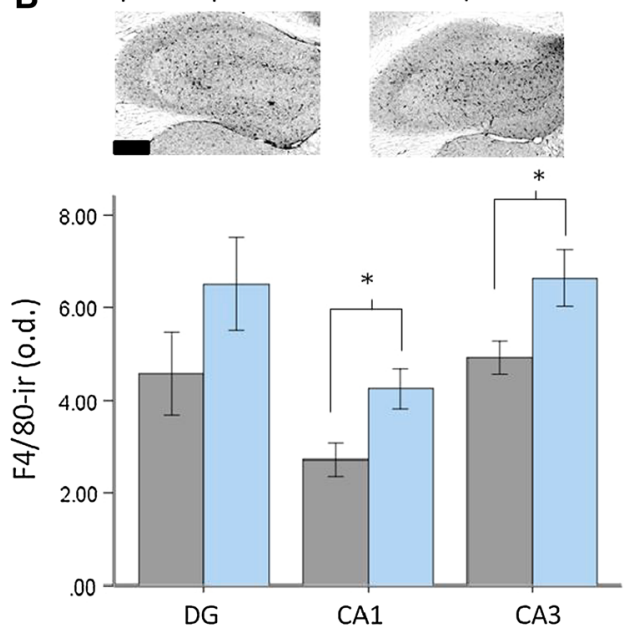

Prior sepsis
A

\section{2 hours after LPS}
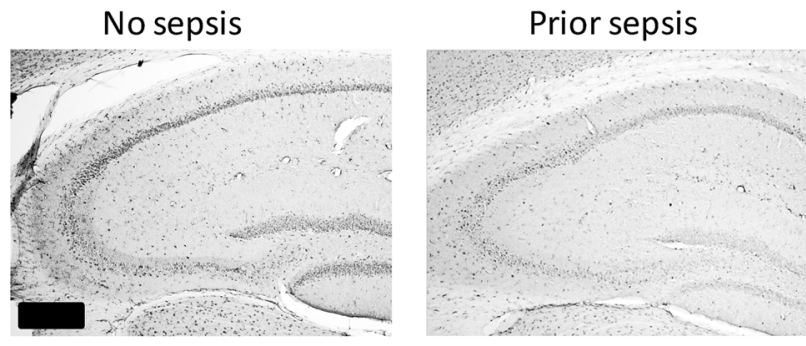

B

$\underline{D G}$
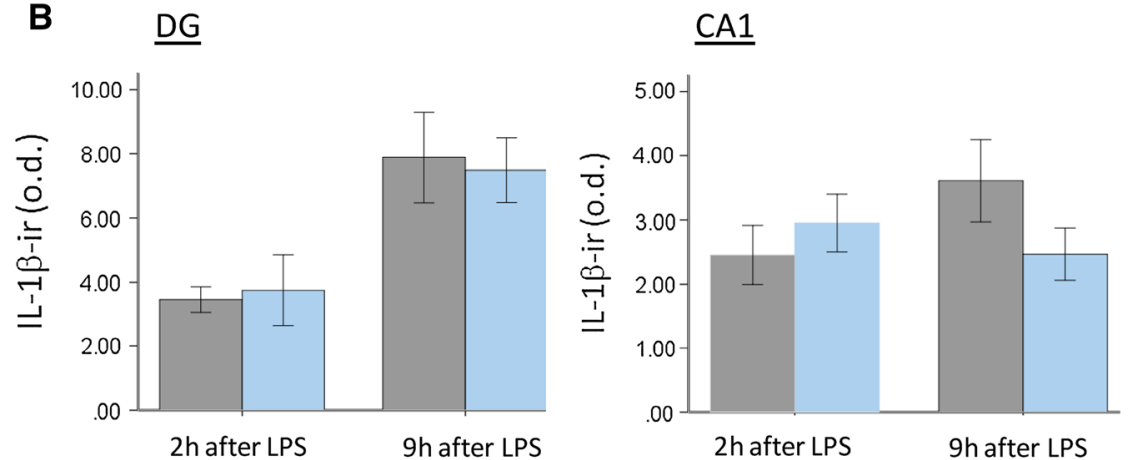

9 hours after LPS
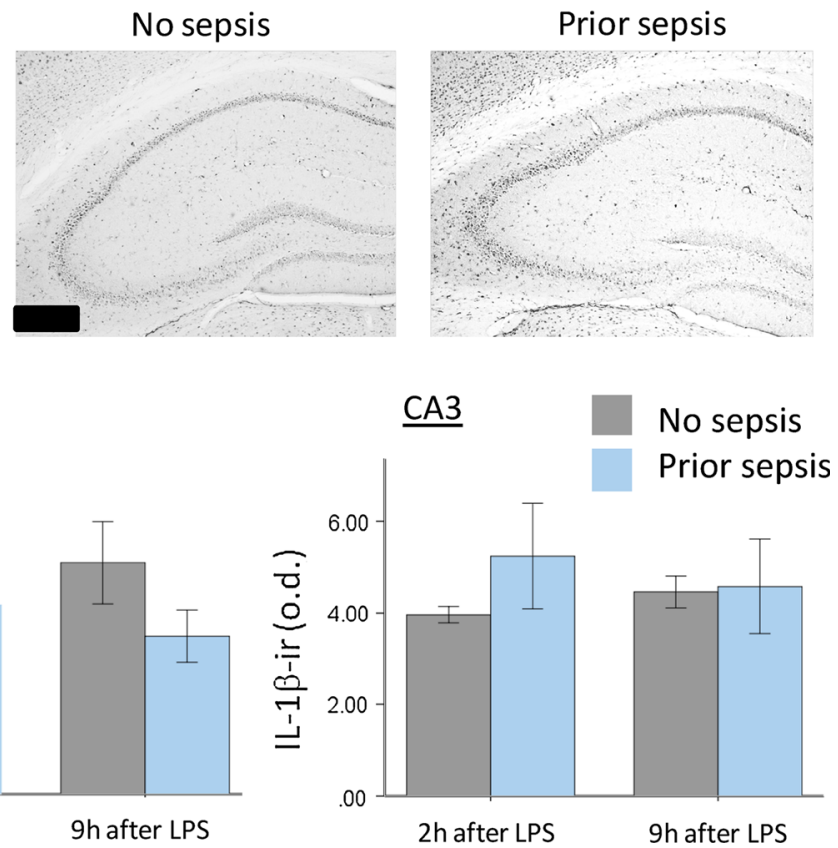

Fig. 4 Expression of IL-1 $\beta$ in the hippocampus following LPS treatment of both control and post-septic animals. a Sample photomicrographs of IL-1 $\beta$ immunostaining in the hippocampus of control and post-septic mice 2 and 9 h after LPS treatment. b Quantification of IL-1 $\beta$ immunstaining in hippocampal subfields. $N=4$ per group

previously undergone sepsis that there are persistent changes in neurophysiological, cognitive and behavioural parameters (Calsavara et al. 2013; Iwashyna et al. 2010; Weberpals et al. 2009; O'Callaghan et al. 2012). These changes appear to model the syndrome of post-septic encephalopathy in patients who have survived sepsis and who display cognitive impairments after discharge from hospital when compared to patients who have spent similar durations in intensive care wards due to other conditions (Semmler et al. 2013; Siami 

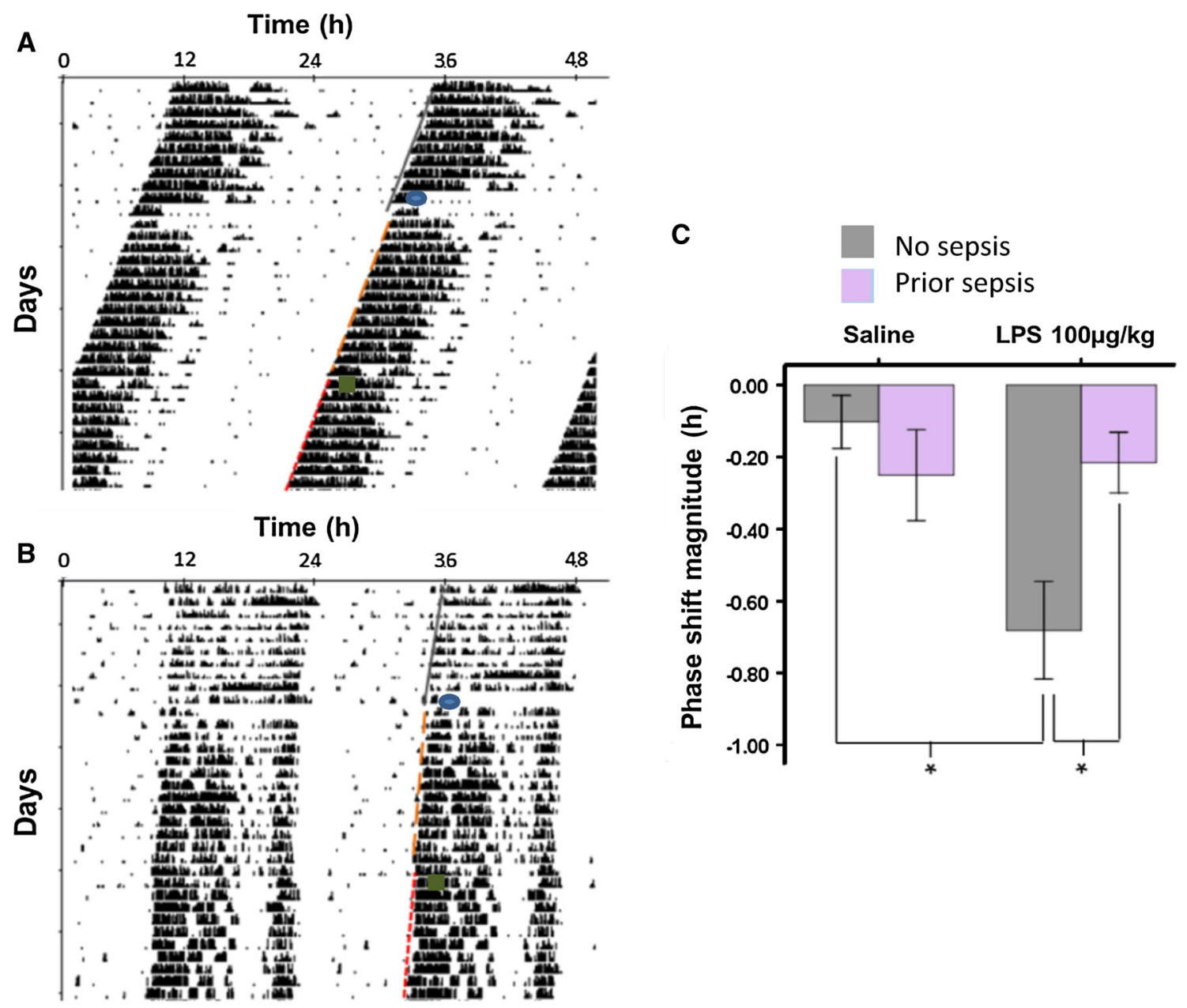

Fig. 5 Circadian phase shifts in response to LPS treatment in control and post-septic mice. Sample actograms from a $\mathbf{a}$ control and $\mathbf{b}$ a post-septic animal showing the free running rhythm in DD before and after challenge with both LPS (blue circle) and saline (green square). The imposed lines show the lines of best fit through the activity onsets

et al. 2008; Iwashyna et al. 2010). Animal studies of models of neurodegeneration have indicated that the ongoing process of neuronal cell death, coupled with concomitant neuroinflammation, primes microglia for exaggerated responses to peripheral immune challenge and leads to more exacerbated behavioural and cognitive effects than would normally be elicited by such an immune challenge against a normal neuroimmunological background (reviewed by Cunningham 2013). Further, immune challenge in healthy older animals leads to an exaggerated behavioural response, and aging in associated with increased neuroinflammation (Norden and Godbout 2013). Therefore we set out to examine whether the neuroinflammation observed postsepsis in the mouse might be associated with altered responses to LPS.

The open field test is a mainstay of assessing sickness behaviour that follows from peripheral immune challenge, prior to and after each treatment. c Shows the magnitude of the phase delays elicited by both saline and LPS treatment at CT15 in control and post-septic animals. $N=10$ for the control group and $N=12$ for the post-septic group. $* P<0.05$

as it allows for motor activity and exploration to be observed in the same task (Yirmiya et al. 1994). In our study we found the expected suppression by LPS of locomotion and exploration in the OFT, although we found no evidence that prior sepsis affected this. Further, as LPS treatment induces a depressive-like state with a delayed time course compared to sickness behaviour (Dantzer et al. 2008), we tested response to acute LPS on the TST, a well validated model of behavioural despair (Cryan et al. 2005). We observed the expected LPS-induced increase in immobility, but again found no evidence for altered responses to LPS in post-septic animals. It may be that prior sepsis simply does not affect the behavioural expression of subsequent LPS-induced behaviours, or it may be that the acute LPS dose used in the current study $(100 \mu \mathrm{g} / \mathrm{kg})$, which induces a mild to moderate sickness behaviour (Cunningham et al. 2005) is not the optimal dose 

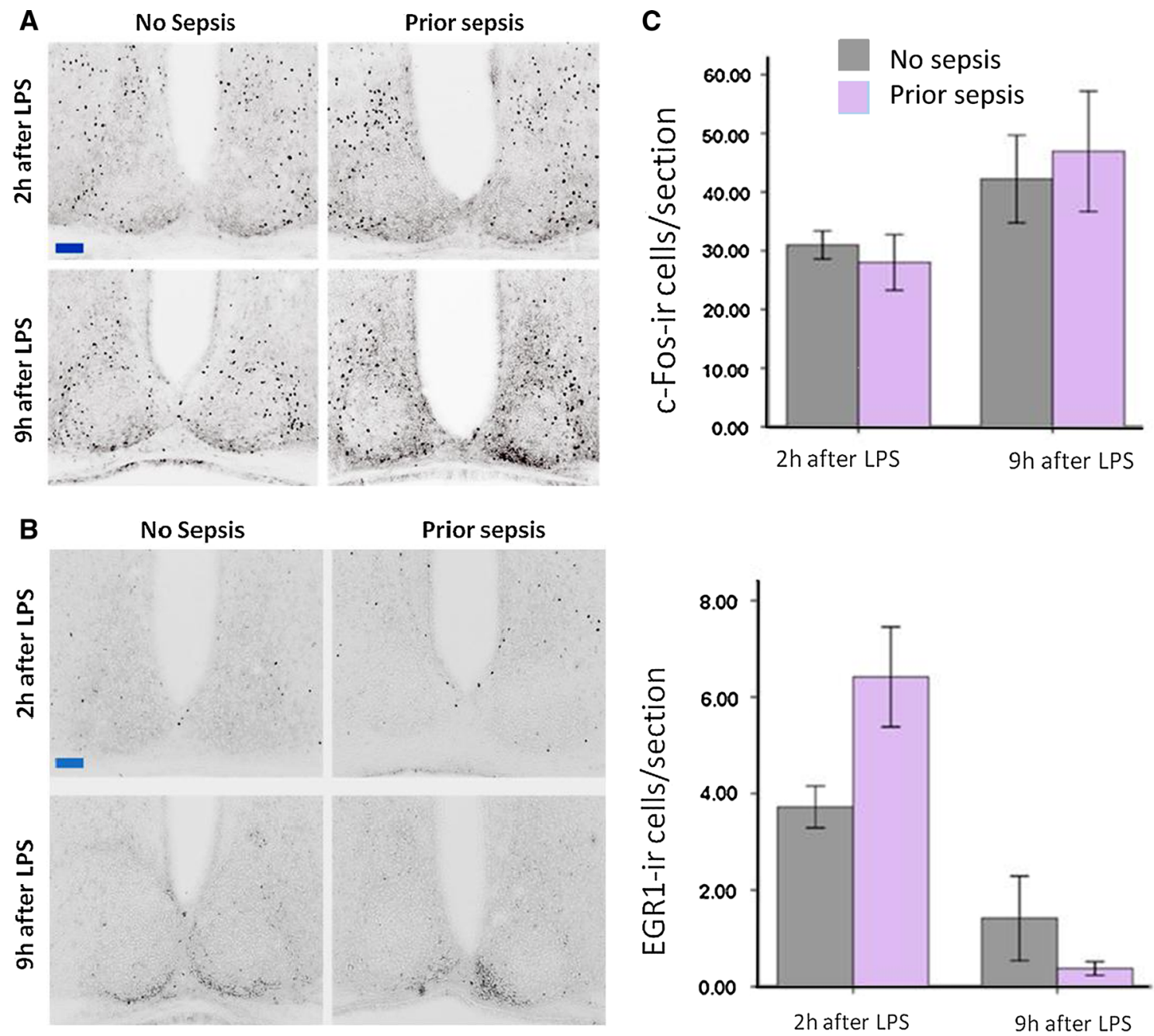

Fig. 6 Effect of prior sepsis on the expression of immediate early gene products in the SCN of control and post-septic animals. a and b Representative photomicrographs showing c-Fos and EGR1 immunostaining respectively in the $\mathrm{SCN}$ in control and post-septic

to use, and that increased sensitivity to a lower dose of LPS may be seen in post-septic animals; future experiments will need to address such issues.

It is also interesting to note that whilst we observed no behavioural impacts of prior sepsis on LPS-induced effects, we did observe some changes in immediate early gene expression, with significant changes in c-Fos and EGR1 expression observed in the hippocampus of post-septic animals following acute LPS treatment compared to control non-sepsis animals treated with LPS. Previous data has shown that forebrain c-Fos expression is induced by acute LPS treatment in the mouse (Frenois et al. 2007) and EGR1 expression in the forebrain is also altered acutely by LPS treatment (Bonow et al. 2009; Gavilán et al. 2009). The functional relevance of altered c-Fos and EGR1 expression in the hippocampus after acute LPS treatment in post-septic animals at 2 and $9 \mathrm{~h}$ after a LPS injection. $N=4-5$ for each group per timepoint. Scale bar $100 \mu \mathrm{m}$. c and d Quantification of c-Fos and EGR1 immunostaining respectively in the SCN at 2 and $9 \mathrm{~h}$ after acute LPS treatment

animals is unclear. EGR1 has been implicated as a plasticity-associated mediator reported to be important in longterm memory (Davis et al. 2003) and it will be of interest in the future to assess acute LPS-induced effects on memory formation and recall in behavioural tasks such as the Morris water maze in post-septic animals, as acute LPS is well described as inducing cognitive deficits in such tasks (Shaw et al. 2001). An interesting precedent is found in the expression of another plasticity-associated immediate early gene product, ARC, whose expression in the dentate gyrus is upregulated in a model of chronic neuroinflammation involving ongoing central injection of LPS, with this upregulation being associated with impaired performance on spatial memory tasks (Hauss-Wegrzyniak et al. 1998; Rosi et al. 2005). The changes in c-Fos and EGR1 expression in the post-septic animals may represent an 
A

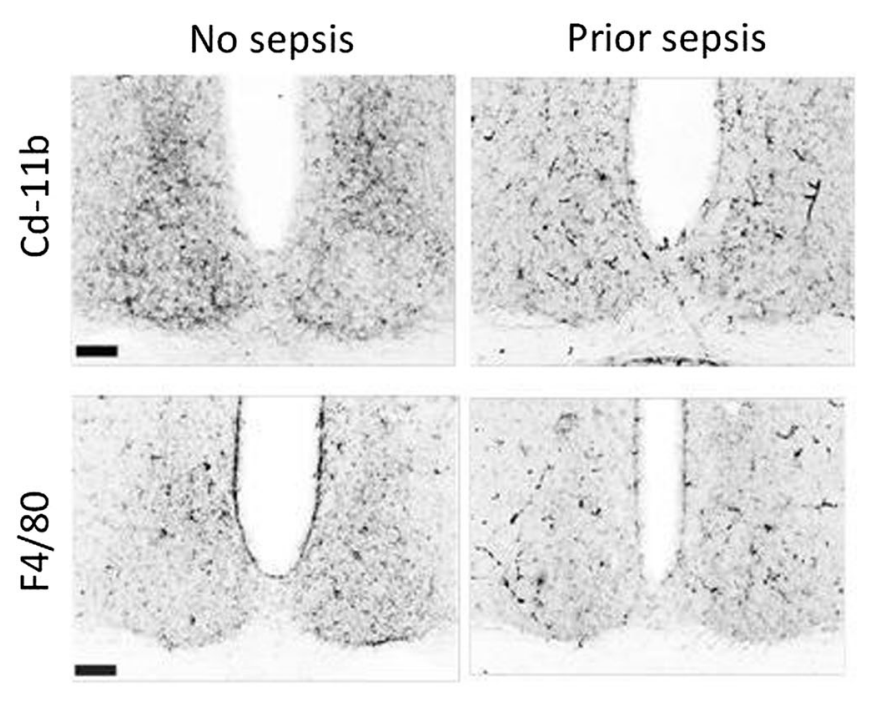

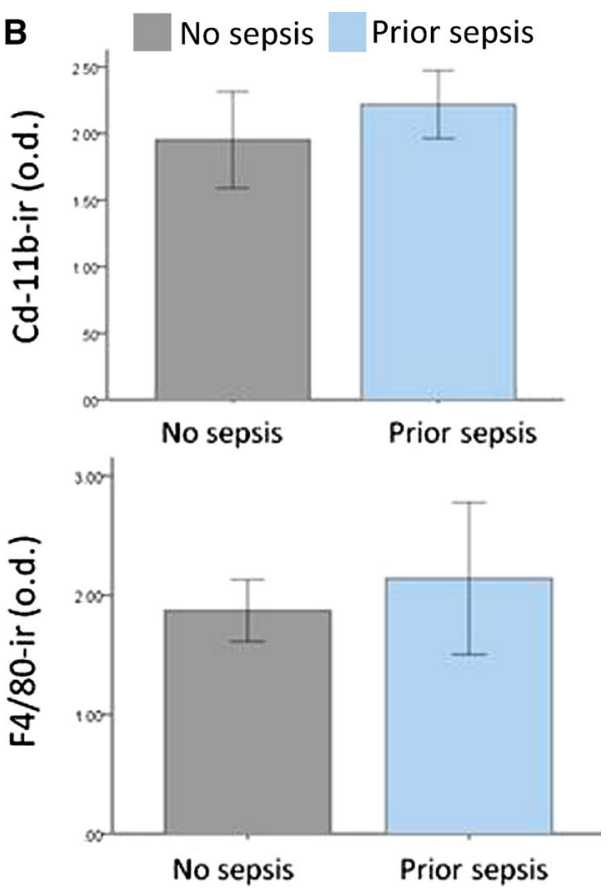

Fig. 7 Expression of Cd-11b and F4/80 in the SCN following LPS treatment of both control and post-septic mice. a Sample photomicrographs and $\mathbf{b}$ quantification of cd-11b and F4/80 immunostaining

increased central sensitivity to LPS-induced systematic immunomediators (e.g. TNF- $\alpha$, IL- $1 \beta$ ), and it would be interesting to test the behavioural and molecular responses of post-septic animals not only to different doses of peripheral LPS, but also to peripheral or central treatment with mediators such as TNF- $\alpha$ and IL-1 $\beta$.

For the analysis of circadian responses to acute LPS treatment, we built on observations that peripheral LPS treatment induces moderate phase delays of the free-running rhythm of locomotor behaviour, but only when LPS is applied at CT15 (in the early portion of the activity phase; Marpegán et al. 2005). This effect of acute LPS is dependent on TLR4 (Paladino et al. 2010) and involves the central action of TNF- $\alpha$ (Leone et al. 2012). Further the $\mathrm{SCN}$ has been shown to be sensitive to peripheral immune challenge, with SCN levels of activated NF- $\mathrm{BB}$ p65, c-Fos and EGR1 acutely-induced by peripheral LPS treatment (Beynon and Coogan 2010; O'Callaghan et al. 2012). Further we have previously described changes in circadian clock resetting in post-septic animals (O'Callaghan et al. 2012). To our surprise we observed that treatment of postseptic animals with acute LPS did not result in any significant phase shift, in comparison to control animals who displayed the expected phase delays. We do not believe that there is an explanation offered for such findings in the alteration of core circadian parameters in post-septic animals, as in the current studies and in previous studies
$4 \mathrm{~h}$ after LPS treatment in control and post-septic animals. $N=5-7$ per group. Scale bar $100 \mu \mathrm{m}$

(O'Callaghan et al. 2012) we do not find alterations in free running period, rhythm power or other core circadian parameters. One possible explanation for the lack of effect of acute LPS effects on the circadian system in post-septic animals is the immunosuppression following from sepsis (Hotchkiss et al. 2013) and/or from endotoxin tolerance (Morris and Li 2012). Such effects seem somewhat unlikely, given that the timeframe between the septic LPS treatment and the shortest time to the acute LPS treatment is 4 weeks, by which time it would LPS-tolerance would be expected to have dissipated (West and Heagy 2002). Further, the results from the open field and tail suspension tests indicate that LPS induces the same levels of behavioural effects in post-septic animals as in controls, suggesting that post-septic mice, at the time of testing used in our experiments, are capable of producing appropriate behavioural responses to further LPS challenge. Therefore it is not clear as to the mechanisms by which there is an altered circadian response to LPS in post-septic animals, and if this effect is specific to the circadian system or is a secondary effect of a more universal change. For example, Weberpals et al. (2009) described changes in cerebral metabolism in postseptic mice, and such changes may have an impact on the response of the SCN to LPS. Further, loss of dendritic spines has been described post-LPS in mice (Kondo et al. 2011), and such a loss might induce a functional rearrangement in the $\mathrm{SCN}$ and different characteristics of phase 
resetting, as a previous study demonstrated altered resetting in post-septic animals (O'Callaghan et al. 2012). There were no apparent differences in the levels of c-Fos or EGR1 in the SCN following on from an acute LPS treatment in post-septic animals, although it should be noted that for this analysis that LPS treatment occurred during the subjective day, and not at CT15 which is the time of treatment used to elicit behavioural phase shifts. We chose this time of treatment as previous work had indicated that acute LPS treatment during the subjective day can upregulate SCN expression of both c-Fos and EGR1 (Beynon and Coogan 2010; O'Callaghan et al. 2012).

The analysis of the microglial markers F4/80 and Cd- $11 \mathrm{~b}$ indicate that there is no difference in their expression in the SCN following acute LPS treatment between post-septic and control animals. However we do report a finding that levels of both these antigens are elevated in the hippocampus following acute LPS treatment in post-septic animals. It may be that microglia are more reactive to subsequent challenge in post-septic animals, and that this effects is region-specific, or it may reflect a general upregulation of microglial markers in the post-septic hippocampus (e.g. Weberpals et al. 2009), or a combination of the two. Our finding that hippocampal IL$1 \beta$ levels are not enhanced may be taken to indicate that microglia are not more responsive, as priming experiments have previously shown upregulation of IL- $1 \beta$ in microglia following LPS treatment against a background of neurodegeneration (Cunningham et al. 2005).

Overall, this study indicates that prior LPS-induced sepsis has some modality-specific effects on behavioural response to subsequent immune challenge. Future work may examine if prior sepsis alters performance on cognitive and a broader range of behavioural tasks following acute immune challenge in order to understand to what extent exaggerated responses to subsequent infections may contribute to the decrement of quality of life associated with post-septic encephalopathy (Iwashyna et al. 2010).

Acknowledgments We acknowledge funding from the Health Research Board and from a John and Pat Hume Scholarship, NUI Maynooth.

Conflict of interest There are no conflicts of interest.

\section{References}

Angus DC, van der Poll T (2013) Severe sepsis and septic shock. N Engl J Med 369:840-851

Bass J, Takahashi JS (2010) Circadian integration of metabolism and energetics. Science 330:1349-1354

Beynon AL, Coogan AN (2010) Diurnal, age, and immune regulation of interleukin-1 $\beta$ and interleukin- 1 type 1 receptor in the mouse suprachiasmatic nucleus. Chronobiol Int 27:1546-1563

Bonow RH, Aïd S, Zhang Y, Becker KG, Bosetti F (2009) The brain expression of genes involved in inflammatory response, the ribosome, and learning and memory is altered by centrally injected lipopolysaccharide in mice. Pharmacogenomics $\mathbf{J}$ 9:116-126

Bossù $\mathrm{P}$, Cutuli D, Palladino I, Caporali $\mathrm{P}$, Angelucci F, Laricchiuta D, Gelfo F, De Bartolo P, Caltagirone C, Petrosini L (2012) A single intraperitoneal injection of endotoxin in rats induces longlasting modifications in behavior and brain protein levels of TNF- $\alpha$ and IL-18. J Neuroinflammation 9:101

Calsavara AC, Rodrigues DH, Miranda AS, Costa PA, Lima CX, Vilela MC, Rachid MA, Teixeira AL (2013) Late anxiety-like behavior and neuroinflammation in mice subjected to sublethal polymicrobial sepsis. Neurotox Res 24:103-108

Chen J, Buchanan JB, Sparkman NL, Godbout JP, Freund GG, Johnson RW (2008) Neuroinflammation and disruption in working memory in aged mice after acute stimulation of the peripheral innate immune system. Brain Behav Immun 22:301-311

Coogan AN, Wyse CA (2008) Neuroimmunology of the circadian clock. Brain Res 1232:104-112

Coogan AN, Schutová B, Husung S, Furczyk K, Baune BT, Kropp P, Häßler F, Thome J (2013) The circadian system in Alzheimer's disease: disturbances, mechanisms, and opportunities. Biol Psychiatry 74:333-339

Cryan JF, Mombereau C, Vassout A (2005) The tail suspension test as a model for assessing antidepressant activity: review of pharmacological and genetic studies in mice. Neurosci Biobehav Rev 29:571-625

Cunningham C (2013) Microglia and neurodegeneration: the role of systemic inflammation. Glia 61:71-90

Cunningham C, Wilcockson DC, Campion S, Lunnon K, Perry VH (2005) Central and systemic endotoxin challenges exacerbate the local inflammatory response and increase neuronal death during chronic neurodegeneration. J Neurosci 25:9275-9284

Dantzer R (2001) Cytokine-induced sickness behavior: mechanisms and implications. Ann N Y Acad Sci 933:222-234

Dantzer R, O'Connor JC, Freund GG, Johnson RW, Kelley KW (2008) From inflammation to sickness and depression: when the immune system subjugates the brain. Nat Rev Neurosci 9:46-56

Davis S, Bozon B, Laroche S (2003) How necessary is the activation of the immediate early gene zif268 in synaptic plasticity and learning? Behav Brain Res 142:17-30

Dibner C, Schibler U, Albrecht U (2010) The mammalian circadian timing system: organization and coordination of central and peripheral clocks. Annu Rev Physiol 72:517-549

Frenois F, Moreau M, O'Connor J, Lawson M, Micon C, Lestage J, Kelley KW, Dantzer R, Castanon N (2007) Lipopolysaccharide induces delayed FosB/DeltaFosB immunostaining within the mouse extended amygdala, hippocampus and hypothalamus, that parallel the expression of depressive-like behavior. Psychoneuroendocrinology 32:516-531

Gavilán MP, Castaño A, Torres M, Portavella M, Caballero C, Jiménez S, García-Martínez A, Parrado J, Vitorica J, Ruano D (2009) Age-related increase in the immunoproteasome content in rat hippocampus: molecular and functional aspects. J Neurochem 108:260-267

Griffin EW, Skelly DT, Murray CL, Cunningham C (2013) Cyclooxygenase-1-dependent prostaglandins mediate susceptibility to systemic inflammation-induced acute cognitive dysfunction. J Neurosci 33:15248-15258

Hauss-Wegrzyniak B, Dobrzanski P, Stoehr JD, Wenk GL (1998) Chronic neuroinflammation in rats reproduces components of the neurobiology of Alzheimer's disease. Brain Res 780:294-303

Henry CJ, Huang Y, Wynne AM, Godbout JP (2009) Peripheral lipopolysaccharide (LPS) challenge promotes microglial hyperactivity in aged mice that is associated with exaggerated induction of both pro-inflammatory IL-1beta and anti-inflammatory IL-10 cytokines. Brain Behav Immun 23:309-317 
Hotchkiss RS, Monneret G, Payen D (2013) Immunosuppression in sepsis: a novel understanding of the disorder and a new therapeutic approach. Lancet Infect Dis 13:260-268

Iwashyna TJ, Ely EW, Smith DM, Langa KM (2010) Long-term cognitive impairment and functional disability among survivors of severe sepsis. JAMA 304:1787-1794

Kondo S, Kohsaka S, Okabe S (2011) Long-term changes of spine dynamics and microglia after transient peripheral immune response triggered by LPS in vivo. Mol Brain 4:27

Leone MJ, Marpegan L, Duhart JM, Golombek DA (2012) Role of proinflammatory cytokines on lipopolysaccharide-induced phase shifts in locomotor activity circadian rhythm. Chronobiol Int 29:715-723

Marpegán L, Bekinschtein TA, Costas MA, Golombek DA (2005) Circadian responses to endotoxin treatment in mice. J Neuroimmunol 160(1-2):102-109

McClung CA (2013) How might circadian rhythms control mood? Let me count the ways. Biol Psychiatry 74:242-249

McCusker RH, Kelley KW (2013) Immune-neural connections: how the immune system's response to infectious agents influences behaviour. J Exp Biol 216:84-98

Mohawk JA, Green CB, Takahashi JS (2012) Central and peripheral circadian clocks in mammals. Annu Rev Neurosci 35:445-462

Morris M, Li L (2012) Molecular mechanisms and pathological consequences of endotoxin tolerance and priming. Arch Immunol Ther Exp (Warsz) 60:13-18

Norden DM, Godbout JP (2013) Review: microglia of the aged brain: primed to be activated and resistant to regulation. Neuropathol Appl Neurobiol 39:19-34

O'Callaghan EK, Anderson ST, Moynagh PN, Coogan AN (2012) Long-lasting effects of sepsis on circadian rhythms in the mouse. PLoS One 7:e47087

Paladino N, Leone MJ, Plano SA, Golombek DA (2010) Paying the circadian toll: the circadian response to LPS injection is dependent on the Toll-like receptor 4. J Neuroimmunol 225:62-67
Qin L, Wu X, Block ML, Liu Y, Breese GR, Hong JS, Knapp DJ, Crews FT (2007) Systemic LPS causes chronic neuroinflammation and progressive neurodegeneration. Glia 55:453-462

Rosi S, Ramirez-Amaya V, Vazdarjanova A, Worley PF, Barnes CA (2005) Wenk GL (2005) Neuroinflammation alters the hippocampal pattern of behaviorally induced Arc expression. J Neurosci 25:723-731

Semmler A, Widmann CN, Okulla T, Urbach H, Kaiser M, Widman G, Mormann F, Weide J, Fliessbach K, Hoeft A, Jessen F, Putensen C, Heneka MT (2013) Persistent cognitive impairment, hippocampal atrophy and EEG changes in sepsis survivors. J Neurol Neurosurg Psychiatry 84:62-69

Shaw KN, Commins S, O'Mara SM (2001) Lipopolysaccharide causes deficits in spatial learning in the watermaze but not in BDNF expression in the rat dentate gyrus. Behav Brain Res 124:47-54

Siami S, Annane D, Sharshar T (2008) The encephalopathy in sepsis. Crit Care Clin 24:67-82

Steru L, Chermat R, Thierry B, Simon P (1985) The tail suspension test: a new method for screening antidepressants in mice. Psychopharmacology 85:367-370

Vilaplana J, Lavialle M (1999) A method to quantify glial fibrillary acidic protein immunoreactivity on the suprachiasmatic nucleus. J Neurosci Methods 88:181-187

Weberpals M, Hermes M, Hermann S, Kummer MP, Terwel D, Semmler A, Berger M, Schäfers M, Heneka MT (2009) NOS2 gene deficiency protects from sepsis-induced long-term cognitive deficits. J Neurosci 29:14177-14184

West MA, Heagy W (2002) Endotoxin tolerance: a review. Crit Care Med 30:S64-S73

Yirmiya R, Rosen H, Donchin O, Ovadia H (1994) Behavioral effects of lipopolysaccharide in rats: involvement of endogenous opioids. Brain Res 648:80-86 